\title{
Cosmological screening and the phantom braneworld model
}

\author{
Sourav Bhattacharya ${ }^{1, \mathrm{a}}$, Stefanos R. Kousvos ${ }^{2, \mathrm{~b}}$, Stylianos Romanopoulos ${ }^{2, \mathrm{c}}$, Theodore N. Tomaras ${ }^{2, \mathrm{~d}}$ \\ ${ }^{1}$ Department of Physics, Indian Institute of Technology Ropar, Rupnagar, Punjab 140 001, India \\ ${ }^{2}$ ITCP and Department of Physics, University of Crete, 70013 Heraklion, Greece
}

Received: 19 June 2018 / Accepted: 31 July 2018 / Published online: 10 August 2018

(C) The Author(s) 2018

\begin{abstract}
The scalar and vector cosmological perturbations at all length scales of our Universe are studied in the framework of the phantom braneworld model. The model is characterized by the parameter $\Omega_{M} \equiv M^{3} / 2 m^{2} H_{0}$, with $M$ and $m$ the five- and four-dimensional Planck scales, respectively, and $H_{0}$ the Hubble parameter today, while $\Omega_{M} \rightarrow 0$ recovers the $\Lambda \mathrm{CDM}$ model. Ignoring the backreaction due to the peculiar velocities and also the bulk cosmological constant, allows the explicit computation of the gravitational potentials, $\Phi$ and $\Psi$. They exhibit exponentially decreasing screening behaviour characterized by a screening length which is a function of the quasidensity parameter $\Omega_{M}$.
\end{abstract}

\section{Introduction}

In the braneworld (BW) model the $3+1$-dimensional Universe we live in is a timelike hypersurface (the brane) of codimension one or more, embedded in a higher dimensional spacetime (the world), see [1,2] for a vast review and also references therein. Unlike the higher dimensional theories such as Gauss-Bonnet gravity, e.g. [3], in the BW model all standard model matter fields are confined on the brane whereas only gravity can propagate in the extra dimension(s).

The existence of the extra dimension implies departure from General Relativity. For example in the RandallSundrum model with a single extra dimension, the modification occurs at the small scales $[4,5]$. The extra dimension needs to neither be small nor compact and can even be infinite. Compact extra dimensions, on the other hand, imply an infinite and discrete Kaluza-Klein spectrum on the brane, see e.g. [6]. We further refer our reader to [7-12] for a description

\footnotetext{
a e-mail: sbhatta@iitrpr.ac.in

be-mail: skousvos@physics.uoc.gr

c e-mail: sromanop@physics.uoc.gr

de-mail: tomaras@physics.uoc.gr
}

of fitting the galaxy rotation curves and the study of gravitational lensing in this model. While the extra dimension is usually taken to be spacelike, we refer our reader to [13] for a timelike extra dimension.

Discussions on static solutions such as a black hole in the BW model can be seen in [14-17] and references therein. For the so called two branch RS-I model, from the modification of Newton's law, the upper bound on the bulk anti-de Sitter radius turns out to be $l \lesssim 14 \mu \mathrm{m}$; whereas for the one branch RS-II model, the binary gravity wave data puts a bound : $l \lesssim 3.9 \mu \mathrm{m}$ [18]. Probing the extra dimensional effects by studying the strong gravitational lensing can be seen in [19]. We refer our reader to [20] for a modification of the RS model with cosmological constants associated with both the bulk and the brane, fine tuned to make the bulk flat. This scenario is in particular helpful to estimate the energy lost by the brane via the Kaluza-Klein gravitons. In [21,22], the effect of brane - bulk energy exchange on cosmology was investigated and a model where our current universe is obtained as a late time attractor was proposed. We further refer our reader to [23] for a vast review and an exhaustive list of references pertaining to gravity and cosmology in the context of the braneworld model.

In this paper, we shall be interested in an extension of the Dvali-Gabadadze-Porrati braneworld (DGP) model [24-27] containing in the action, the 4-dimensional Ricci scalar on the brane, induced by the one loop correction due to the graviton-matter interaction, and the extrinsic curvature of the brane. This model, unlike the Randall-Sundrum case, modifies gravity only beyond a characteristic length scale, depending on the five- and four-dimensional Newton constants. The relevant equation of motion gives rise to two branches of cosmological solutions, both with flat spatial sections, one being self accelerated without requiring any dark energy/cosmological constant, whereas the other branch (the normal branch) requires at least one cosmological constant to accommodate for the current accelerated expansion [28-30]. However, the former was shown to have ghost instability in 
subsequent works [31,32], leaving only the "normal" branch to be a possible alternative to the $\Lambda \mathrm{CDM}$ model.

Furthermore, the equation of state parameter for the effective dark energy source is time dependent, $w(t)$, and turns out to be less than minus one today [33-37]. For a certain range of parameter values, $w(t)$ will reach asymptotically the value -1 (the de Sitter phase). Otherwise, the universe can even leave at some stage the phase of accelerated expansion reentering matter domination, thus evading the so called phantom disaster [38]. Since $w(t)<-1$ in the current epoch, this model is often called "phantom braneworld model". Interestingly, this model indicates that the expansion of our universe was stopped at redshift $z \gtrsim 6$ and 'loitered' there for a long period of time favouring structure formation. Arguments supporting this, based on the observed data of population of the quasistellar objects and supermassive black holes in $6 \lesssim z \lesssim 20$ can be found in [36]. Scalar cosmological perturbation theory in the phantom braneworld model and further details are studied in [39-41], while in [42] the stability analysis of large scale cosmic structures via their size-versus-mass study in the context of the present model and in the presence of a bulk cosmological constant, was performed. We also refer our reader to [43] for constraints on the braneworld model via gravity wave data. See also [44] for phenomenological arguments in the favour of $w(t)<-1$ via the neutrino mass higherarchy.

The braneworld model under study here is assumed to have 'zero thickness' in the extra dimension. Interesting effects however, may arise when one considers a thick brane $[45,46]$. In particular, in such a scenario, with a large extra dimension, one can have a new energy scale on the brane, determined by both brane thickness and the size of the extra dimension. For energies much larger than this new scale, the physics in the brane depends upon the position along the extra dimension, while for much smaller energies the equivalence principle may be violated, resulting in certain fine tuning to preserve it.

Given that the phantom braneworld model modifies gravity significantly at large scales, it becomes an interesting task to investigate this model's prediction at arbitrarily large distances. One such arena seems to be the study of screening effects, where certain terms in the scalar perturbation equation, which we can ignore at small scales, lead to modifications of the gravitational potential at large scales [47-57]. By approximating the inhomogeneities of our universe as delta function sources, a first order analytical formalism for the cosmological scalar and vector perturbations for the $\Lambda \mathrm{CDM}$ model was developed recently in [47], where a Yukawa-like fall-off of the gravitational potential was derived at large scales. Various extensions of this work, including the case of interacting fluid sources, can be found in [48-53]. Discussions on the $N$-body simulations in the context of cosmic screening can be seen in $[54,55]$. We further refer our reader to $[56,57]$ for second order computations on the scalar perturbation pertaining respectively to the $\Lambda \mathrm{CDM}$ and the Einstein de Sitter models. The extra dimensional scenario is certainly not included in the above examples. Motivated by this, we shall study in this work the first order cosmological screening in the phantom braneworld model. Our chief goal would be, apart from casting the perturbation equations in a suitable form and solving them, to point out differences of this model from $\triangle C D M$, that can arise at very large scales.

The paper is organized as follows. In the next section we briefly review the phantom braneworld model. In Sect. 3 we develop the first order equations pertaining to the scalar and the vector perturbations with no bulk cosmological constant. In Sect. 4 we solve for the scalar perturbation ignoring the peculiar velocities, and compare it both analytically and numerically with the $\Lambda \mathrm{CDM}$ model. We conclude with a Sect. 5 .

We shall use mostly negative signature for the metric and will set $c=1$ throughout.

\section{The phantom braneworld model}

Let us first briefly review the basic features of the phantom braneworld model, details of which can be seen in e.g. [40] and references therein. The relevant action is given by,

$$
\begin{aligned}
S= & M^{3}\left[\int_{\text {bulk }}\left(\mathcal{R}-2 \Lambda_{5 D}\right)-2 \int_{\text {brane }} K\right]+\int_{\text {brane }}\left(m^{2} R-2 \sigma\right) \\
& -\int_{\text {brane }} L\left(g_{\mu \nu}, \phi\right)
\end{aligned}
$$

where $\mathcal{R}$ and $R$ are the Ricci scalars corresponding to five (the bulk) and four dimensions (the brane) and $M$ and $m$ are the respective Planck masses. The quantity $\Lambda_{5 D}$ is the cosmological constant in the bulk and $\sigma$ is the brane tension, related to the brane cosmological constant $\Lambda$ by $\Lambda=\sigma / \mathrm{m}^{2} . K$ is the trace of the extrinsic curvature of the brane. $L\left(g_{\mu \nu}, \phi\right)$ stands collectively for all matter fields, $\phi$, confined to the brane and $g_{\mu \nu}$ is the induced metric on it. For our current purpose, $\phi$ would correspond only to the cold dark matter.

Being interested in the $3+1$-dimensional physics, we choose to measure energies in units of the four-dimensional Planck mass $m$. So, we set $m=1$ throughout.

Using the Gauss-Codacci relations, the Einstein equations on the brane become

$$
\begin{aligned}
& G_{\mu \nu}-\left(\frac{\Lambda_{R S}}{b+1}\right) g_{\mu \nu} \\
& \quad=\left(\frac{b}{b+1}\right) T_{\mu \nu}-\left(\frac{1}{b+1}\right)\left[\frac{1}{M^{6}} Q_{\mu \nu}-\mathcal{C}_{\mu \nu}\right]
\end{aligned}
$$

where

$b=\frac{1}{6} \Lambda l^{2}, \quad l=\frac{2}{M^{3}}, \quad \Lambda_{R S}=\frac{\Lambda_{5 D}}{2}+\frac{1}{12} \Lambda^{2} l^{2}$ 
are convenient parameters, and

$$
\begin{aligned}
Q_{\mu \nu}= & \frac{1}{3} E E_{\mu \nu}-E_{\mu \lambda} E_{\nu}^{\lambda}+\frac{1}{2}\left(E_{\rho \lambda} E^{\rho \lambda}-\frac{1}{3} E^{2}\right) g_{\mu \nu}, \\
& E_{\mu \nu} \equiv G_{\mu \nu}-T_{\mu \nu}, \quad E=E_{\mu}^{\mu}
\end{aligned}
$$

The tensor $\mathcal{C}_{\mu \nu}$ is traceless, coming from the projection of the five-dimensional Weyl tensor onto the brane. Taking the divergence of Eq. (2) yields the constraint equation,

$$
\nabla^{\mu}\left(\frac{b+1}{m^{2}} E_{\mu \nu}+T_{\mu \nu}+\frac{1}{M^{6}} Q_{\mu \nu}-\mathcal{C}_{\mu \nu}\right)=0
$$

The spatially homogeneous Einstein equation reads (in conformal time, $\eta$ ) with the cold dark matter as the source,

$$
\begin{aligned}
\frac{\mathcal{H}^{2}}{a^{2}}= & \frac{\bar{\rho}}{3 a^{3}}+\frac{\Lambda}{3} \\
& +\frac{2}{l^{2}}\left[1-\sqrt{1+l^{2}\left(\frac{\bar{\rho}}{3 a^{3}}+\frac{\Lambda}{3}-\frac{\Lambda_{5 D}}{6}-\frac{C}{a^{4}}\right)}\right]
\end{aligned}
$$

where $a \equiv a(\eta)$ is the scale factor, $\mathcal{H}=a^{-1} d a / d \eta$ is the Hubble rate and $\bar{\rho}$ the time independent background homogeneous cold dark matter density in co-moving coordinates. The constant $C$ is due to the existence of the Weyl tensor in the bulk. Due to the radiation like behavior of the term containing $C$, it is often named "Weyl radiation". We shall ignore its backreaction effects onto the cosmological background, though we shall take into account the inhomogeneous perturbations of the projection of the Weyl tensor. We will also ignore the backreaction effects of $\Lambda_{5 D}$. Taking $\Omega_{M} \rightarrow 0$ in the above equation one recovers the $\Lambda \mathrm{CDM}$ limit. Notice that Eq. (6) in the absence of $\Lambda_{5 D}$ and $C$ may be conveniently expressed as

$\frac{\mathcal{H}}{a}=\frac{1}{l}\left[\sqrt{1+\frac{l^{2}}{3}\left(\frac{\bar{\rho}}{a^{3}}+\Lambda\right)}-1\right]$

We will also need the derivative of this equation with respect to conformal time $\eta$

$\frac{d \mathcal{H}}{d \eta}=\mathcal{H}^{2}\left(1-\frac{3 \Omega_{m}}{2\left(1+\Omega_{M}\right)}\right)$

where $^{1}$

$\Omega_{m}=\frac{\bar{\rho}}{3 \mathcal{H}^{2} a}, \quad \Omega_{M}=\frac{a M^{3}}{2 \mathcal{H}}, \quad \Omega_{\sigma}=\frac{\Lambda}{3 \mathcal{H}^{2}}$

Consequently, Eq. (6) takes the simple form

$\Omega_{m}+\Omega_{\sigma}-2 \Omega_{M}=1$

in everything that follows we have replaced $\bar{\rho}$ in favor of $3 \mathcal{H}^{2} \Omega_{m} a, M^{3}$ in favor of $2 \Omega_{M} \mathcal{H} / a$ and $\Lambda$ in favor of

\footnotetext{
$\overline{1}$ We would like to mention that the quantity $\Omega_{M}$ is often defined as $\sqrt{\Omega_{l}}$ in the related literature.
}

$3 \mathcal{H}^{2} \Omega_{\sigma}$. It is very convenient since everything we derive may be expressed as functions of $\Omega_{m}$ and $\Omega_{M}$, only $\left(\Omega_{\sigma}\right.$ is solved for from Eq. 10). The advantage of this procedure is twofold, firstly these parameters are dimensionless and we claim rather intuitive to handle, secondly these will make comparison to $\Lambda$ CDM trivial by simply taking $\Omega_{M}$ to zero.

\section{Derivation of scalar and vector perturbation equations}

We shall extend below the linear perturbation scheme developed for the $\Lambda$ CDM model in [47] to the phantom braneworld model described in the preceeding section. We start with the ansatz for the first order McVittie metric on the brane in the Cartesian coordinates,

$$
\begin{aligned}
d s^{2}= & a^{2}(\eta)\left[(1+2 \Phi(\eta, \boldsymbol{x})) d \eta^{2}+2 B_{i}(\eta, \boldsymbol{x}) d \eta d x^{i}\right. \\
& \left.-(1-2 \Psi(\eta, \boldsymbol{x})) \delta_{i j} d x^{i} d x^{j}\right]
\end{aligned}
$$

where $\Phi, \Psi$ and $B_{i}$ 's are respectively the scalar and vector perturbations and the bold font is used to indicate a vector, which determines the position in space where the potentials are evaluated at. Note that unlike the $\Lambda \mathrm{CDM}, \Phi \neq \Psi$ here, owing to the anisotropic stresses originating from the bulk, e.g. [40].

We shall consider the backreaction effects due to $N$ self gravitating moving point masses. Following [47], we define the proper interval for the $n$-th mass,

$d s_{n}=a(\eta)\left[(1+2 \Phi)+2 B_{i} v_{n}^{i}-(1-2 \Psi) \delta_{i j} v_{n}^{i} v_{n}^{j}\right]^{1 / 2} d \eta$

The peculiar velocities appearing above can be evaluated by subtracting from the observed velocity of the mass, the velocity due to the Hubble flow, e.g. [38].

The energy momentum tensor for these point masses is then given by

$T^{\mu \nu}=\sum_{n} \frac{m_{n}}{\sqrt{-g}} \frac{d x_{n}^{\mu}}{d \eta} \frac{d x_{n}^{v}}{d \eta} \frac{d \eta}{d s_{n}} \delta^{3}\left(\boldsymbol{x}-\boldsymbol{x}_{n}\right)$

where $\boldsymbol{x}_{n}$ is the value of the $x$ coordinate (as defined in the metric Eq. 11) where the $n$th particle is located at. Existing data shows that the peculiar velocities are in general rather small or non-relativistic, at most of the order of $10^{6} \mathrm{~ms}^{-1}$ [58]. Putting these all in together, we find from Eq. (13) the energy momentum tensor up to the first order,

$T^{\mu \nu}=\frac{1}{a^{5}}\left(\begin{array}{cc}(1-2 \Phi+3 \Psi) \rho & \sum_{n} \rho_{n} v_{n} \\ \sum_{n} \rho_{n} v_{n} & 0\end{array}\right)$

where each $\rho_{n}$ corresponds to a delta function point mass located at $\boldsymbol{r}_{n}$,

$\rho_{n} \equiv m_{n} \delta^{3}\left(\boldsymbol{x}-\boldsymbol{x}_{n}\right)$ 
We decompose the total energy density $\rho$ in Eq. (14) as,

$\rho=\bar{\rho}+\delta \rho(\eta, \boldsymbol{x}), \quad \bar{\rho}=\sum_{n} m_{n} / V$

where $\delta \rho(\eta, \boldsymbol{x})$ stands for the contribution of the inhomogeneities. The index $n$ runs over all $N$ particles in the Universe. Note here that $\delta \rho$ is not treated as a perturbation, due to the fact that it is dominant at small scales (see [60]).

Since we must have $|\Psi|,|\Phi| \ll 1$ in Eq. (11), we write from Eq. (14) at first order,

$$
\begin{aligned}
\delta T_{00} & =\frac{1}{a}(\delta \rho+2 \bar{\rho} \Phi+3 \bar{\rho} \Psi), \\
\delta T_{0 i} & =\frac{1}{a}\left(\bar{\rho} B_{i}-\sum_{n} \rho_{n} v_{n}^{i}\right), \quad \delta T_{i j}=0
\end{aligned}
$$

the geodesic equation for the nth particle in Eq. (12) also reads,

$$
\left(\left.a \boldsymbol{B}\right|_{\boldsymbol{x}=\boldsymbol{x}_{n}}-a \boldsymbol{v}_{n}\right)^{\prime}=\left.a \nabla \Phi\right|_{\boldsymbol{x}=\boldsymbol{x}_{n}}
$$

where the 'prime' denotes differentiation once with respect to the conformal time $\eta$ and the variations $\delta T_{\mu \nu}, \delta \mathcal{C}_{\mu \nu}$ and $\delta Q_{\mu \nu}$ depend on both space and time. Since we wish to build a perturbation scheme valid all the way to superhorizon scales, we cannot assume that the perturbations' spatial variations dominate over the temporal ones, unlike the case of the study of cosmic structures, e.g. [38].

Finally, we come to the perturbation of the Weyl tensor's projection onto the brane, $\delta \mathcal{C}_{\mu \nu}$. Its most generic form is given by, e.g. [40],

$\delta \mathcal{C}_{\mu \nu}=\frac{1}{a^{2}}\left(\begin{array}{lc}\delta \rho_{\mathcal{C}} & \partial_{i} v_{\mathcal{C}} \\ \partial_{i} v_{\mathcal{C}} & \frac{\delta \rho_{\mathcal{C}}}{3} \delta_{i j}-\delta \pi_{i j}\end{array}\right)$

where $\delta \pi_{i j}=\left(\nabla_{i} \nabla_{j}-g_{i j} \Delta / 3\right) \delta \pi_{\mathcal{C}}(\Delta$ stands for the Euclidean 3-Laplacian) is trace free and $\delta \rho_{\mathcal{C}}, v_{\mathcal{C}}$ and $\delta \pi_{\mathcal{C}}$ are scalars. In particular, $v_{\mathcal{C}}$ can be regarded as a momentum potential, whose backreaction effects will also be ignored, while considering its time evolution also negligible.

The Einstein equations on the brane (Eq. 2), at first order read, after using Eqs. $(17,19)$,

$$
\begin{gathered}
\Delta \Psi-\frac{9 \mathcal{H}^{2} \Omega_{m}}{2 m_{\mathrm{eff}}^{2}} \Psi-3 \mathcal{H} \Psi^{\prime}-3 \mathcal{H}^{2} \Phi \\
=\frac{\delta \rho}{2 m_{\mathrm{eff}}^{2} a}+\frac{\Omega_{M}}{2 m_{\mathrm{eff}}^{2} a^{2}} \delta \rho_{\mathcal{C}}
\end{gathered}
$$

which is the 00 component, and for $i \neq j$

$$
\begin{aligned}
& \frac{\Omega_{M}}{m_{\mathrm{eff}}^{2} a^{2}} \delta \pi_{i j}-\left(1-\frac{3 \Omega_{m}}{4 m_{\mathrm{eff}}^{4}}\right) \\
& \quad \times\left(\partial_{i} \partial_{j}(\Phi-\Psi)-\frac{1}{2} \partial_{(i}\left(B_{j)}^{\prime}+2 \mathcal{H} B_{j)}\right)\right)=0
\end{aligned}
$$

We also have for the vector perturbation,

$$
\frac{1}{4} \Delta B_{i}-\frac{3 \mathcal{H}^{2} \Omega_{m}}{2 m_{\mathrm{eff}}^{2}} B_{i}+\partial_{i}\left(\Psi^{\prime}+\mathcal{H} \Phi\right)=-\frac{1}{2 m_{\mathrm{eff}}^{2} a} \sum_{n} \rho_{n} v_{n}^{i}
$$

where $\Delta$ as earlier is the Laplacian on the Euclidean 3-space and also the function $m_{\text {eff }} \equiv m_{\text {eff }}(\eta)$ has been introduced

$m_{\text {eff }}^{2} \equiv 1+\Omega_{M}$

The $\Lambda$ CDM limit in the above equations is obtained by letting $\Omega_{M} \rightarrow 0$ in which case we recover the results of [47].

At small length scales relevant to cosmic structures, the spatial derivatives of the potential in Eq. (20) dominate over its temporal derivatives and the other effective mass-like terms appearing on the left hand side. Accordingly, at such small scales, Eq. (20) reduces to the Poisson equation, yielding a gravitational potential falling off as $1 / r$, along with a modified Newton's constant [42]. For $\Lambda \mathrm{CDM}$ in particular, we have $\delta \rho_{\mathcal{C}}=0$, yielding Newton's potential. However, at length scales much larger than those of cosmic structures, the temporal derivative and the effective mass terms can be comparable and, as we will show in Sect. 4, this leads to a significant modification in the behavior of the solution of Eq. (20), as is expected due to the presence of the mass-like term on its left-hand side.

The divergence of Eq. (22), in the Poisson gauge $\partial_{i} B_{i}=0$, gives

$$
\Delta \Xi=\partial_{i}\left(\sum_{n} \rho_{n} v_{n}^{i}\right)
$$

where $\Xi:=-2 m_{\mathrm{eff}}^{2} a\left(\Psi^{\prime}+\mathcal{H} \Phi\right)$. The solution of Eq. (24) is

$\Xi=\frac{1}{4 \pi} \sum_{n} m_{n} \frac{\left(\boldsymbol{x}-\boldsymbol{x}_{n}\right) \cdot \boldsymbol{v}_{n}}{\left|\boldsymbol{x}-\boldsymbol{x}_{n}\right|^{3}}$

Equation (22) has the same form as the corresponding solved in [47].

In this work, we are chiefly interested in distinguishing the phantom braneworld model from $\Lambda \mathrm{CDM}$ with respect to the cosmological screening, which is certainly impossible unless we go to very large length scales. Note that at such scales, the backreaction effects due to the peculiar velocities, which are essentially non-relativistic, would be negligible, e.g. [58]. Thus for our current purpose, we shall from now on ignore the peculiar velocities (and hence the vector perturbation) throughout. 


\section{Solutions ignoring peculiar velocities}

From the definition of $\Xi$ and Eq. (25) in the limit of irrelevant peculiar velocities we have (since $\Xi=0$ )

$\Psi^{\prime}=-\mathcal{H} \Phi$

With this equation in hand we can simplify Eq. (20)

$$
\Delta \Psi-\frac{9 \mathcal{H}^{2} \Omega_{m}}{2 m_{\mathrm{eff}}^{2}} \Psi=\frac{\delta \rho}{2 m_{\mathrm{eff}}^{2} a}+\frac{\Omega_{M}}{2 m_{\mathrm{eff}}^{2} a^{2}} \delta \rho_{\mathcal{C}}
$$

On the other hand we can write Eq. (21)

$\frac{\Omega_{M}}{m_{\mathrm{eff}}^{2} a^{2}} \delta \pi_{\mathcal{C}}=\left(1-\frac{3 \Omega_{m}}{4 m_{\mathrm{eff}}^{4}}\right)(\Phi-\Psi)+$ constant

and recall that in a marginally closed universe with a vanishing bulk cosmological constant, one has [39],

$\Delta \delta \pi_{\mathcal{C}}=\frac{\delta \rho_{\mathcal{C}}}{2}$

Combining Eqs. (28) and (29) to eliminate $\delta \pi_{\mathcal{C}}$ we get

$\frac{\Omega_{M}}{2 m_{\mathrm{eff}}^{2} a^{2}} \delta \rho_{\mathcal{C}}=\left(1-\frac{3 \Omega_{m}}{4 m_{\mathrm{eff}}^{4}}\right) \Delta(\Phi-\Psi)$

In order to solve for $\Phi$ and $\Psi$ we want one more equation. This comes from the spatial component of Eq. (5), after using Eqs. (18) and (27), we obtain

$$
\begin{aligned}
& \frac{\Omega_{M}}{2 m_{\mathrm{eff}}^{2} a^{2}}\left(1-\frac{3 \Omega_{m}}{2 m_{\mathrm{eff}}^{2}}\right) \delta \rho \mathcal{C} \\
& =\Delta \Phi-\left(1+\frac{3 \Omega_{m}}{2 m_{\mathrm{eff}}^{4}}\right) \Delta \Psi+\text { constant }
\end{aligned}
$$

We can substitute Eq. (30) into Eqs. (27) and 31 to obtain a system of two equations with only two unknowns, the perturbations $\Phi$ and $\Psi$. The constant in Eqs. (28) and (31) has to be zero in order for the potential to be vanishing at infinity. The solution of the system is straightforward

$$
\Delta \Phi=I \Delta \Psi
$$

and

$$
\Delta \Psi-\frac{9 \mathcal{H}^{2} \Omega_{m, \mathrm{eff}}}{2} \Psi=\frac{\delta \rho}{2 m_{\mathrm{eff}, \Psi}^{2} a}
$$

where

$$
\begin{gathered}
I \equiv 1+\frac{4 \Omega_{M}}{1+2 m_{\mathrm{eff}}^{2}-\frac{3 \Omega_{m}}{2 m_{\mathrm{eff}}^{4}}}, \quad \Omega_{m, \mathrm{eff}} \equiv \frac{\Omega_{m}}{m_{\mathrm{eff}, \Psi}^{2}}, \\
m_{\mathrm{eff}, \Psi}^{2} \equiv m_{\mathrm{eff}}^{2}+(1-I)\left(m_{\mathrm{eff}}^{2}-\frac{3 \Omega_{m}}{4 m_{\mathrm{eff}}^{2}}\right)
\end{gathered}
$$

Equation (33) is identical to the one obtained for $\Lambda \mathrm{CDM}$ derived in [47] if we drop the eff subscripts, it is trivial to solve our equation by comparing with [47], and using eff subscripts wherever appropriate. The solution is

$$
\left.\Psi\right|_{\text {many particle }}=\frac{1}{3}-\frac{1}{8 \pi m_{\mathrm{eff}, \Psi}^{2} a} \sum_{n} \frac{m_{n}}{\left|\boldsymbol{x}-\boldsymbol{x}_{n}\right|} e^{-\frac{\left|\boldsymbol{x}-\boldsymbol{x}_{n}\right|}{\lambda}}
$$

where

$$
\lambda=\sqrt{\frac{2}{9 \mathcal{H}^{2} \Omega_{m, \text { eff }}}}
$$

For a single particle - a single central over-density - the solution for the potential $\Psi$, valid for all length scales is

$\left.\Psi\right|_{\text {one particle }}=-\frac{1}{8 \pi m_{\mathrm{eff}, \Psi}^{2} a} \frac{m_{0}}{r} e^{-r / \lambda}$

where $m_{0}$ is the mass of the central overdensity and $r=|\mathbf{x}|$. We have dropped the $\frac{1}{3}$ which is generated by the existence of an infinite number of point particles. We will prove that this occurs naturally when considering an isolated sub-region of the Universe (e.g. the observable Universe) at the end of this section. The exponential appearing above clearly indicates the suppression of Newton's potential at large scales, originating from the term present in the perturbation equation behaving as an effective mass. Thus the length scale, $\lambda$, should be interpreted as a screening length.

In every case we can find $\Phi$ solving Eq. (32)

$\Phi=I \Psi$

Figures 1, 2 and 3 elucidate various properties of the gravitational potentials and the screening length. We use the values for cosmological parameters $\Omega_{m}=0.3089$ and $H_{0}=67.74 \mathrm{~km} \mathrm{~s}^{-1} \mathrm{Mpc}^{-1}$ as specified in [59] and we examine the gravitational potentials for one particle with mass $M_{\odot}=1.989 \cdot 10^{30} \mathrm{~kg}$. Figure 1 depicts the behavior of the effective mass density parameter and the screening length versus $\Omega_{M}$. The $\Lambda$ CDM limit is obtained by letting $\Omega_{M} \rightarrow 0$.

We also note that since the screening length is typically of the order of $\mathcal{O}\left(10^{3}\right) \mathrm{Mpc}$ (Fig. 1), at length scales comparable of the size of a typical cosmic structure i.e. $\mathcal{O}(100) \mathrm{Mpc}$, Eq. (37) recovers the $1 / r$ fall-off of the gravitational potentials. However, the $1 / m_{\mathrm{eff}, \Phi}^{2} \equiv I / m_{\mathrm{eff}, \Psi}^{2}$ and $1 / m_{\mathrm{eff}, \Psi}^{2}$ terms present modify Newton's 'constant' in $\Phi$ and $\Psi$ respectively and make it time dependent, as discussed in [42].

Figure 2 depicts the behavior of the effective Newton's constant for $\Psi$ and $\Phi$. In the $\Omega_{M} \rightarrow 0$ limit both of them aproach 1 recovering the $\Lambda \mathrm{CDM}$ limit. Note also that in this limit setting further $\bar{\rho} \rightarrow 0\left(\Omega_{m} \rightarrow 0\right)$ removes the exponential fall off since then $\lambda \rightarrow \infty$ (cf., Eqs. 34, 36), yielding Newton's potential for a point mass located in a de Sitter universe. It is easy to verify that, as expected, this is the linearized approximation of the Schwarzschild-de Sitter metric in the McVittie coordinate frame. Similar conclusions hold 

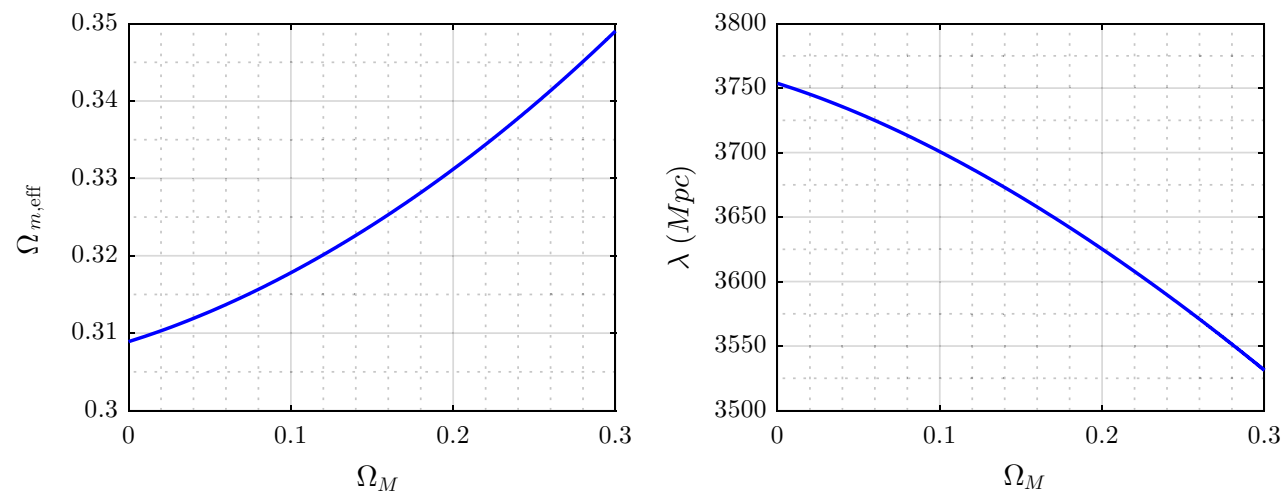

Fig. 1 Plots of the effective mass density and the screening length versus $\Omega_{M}$
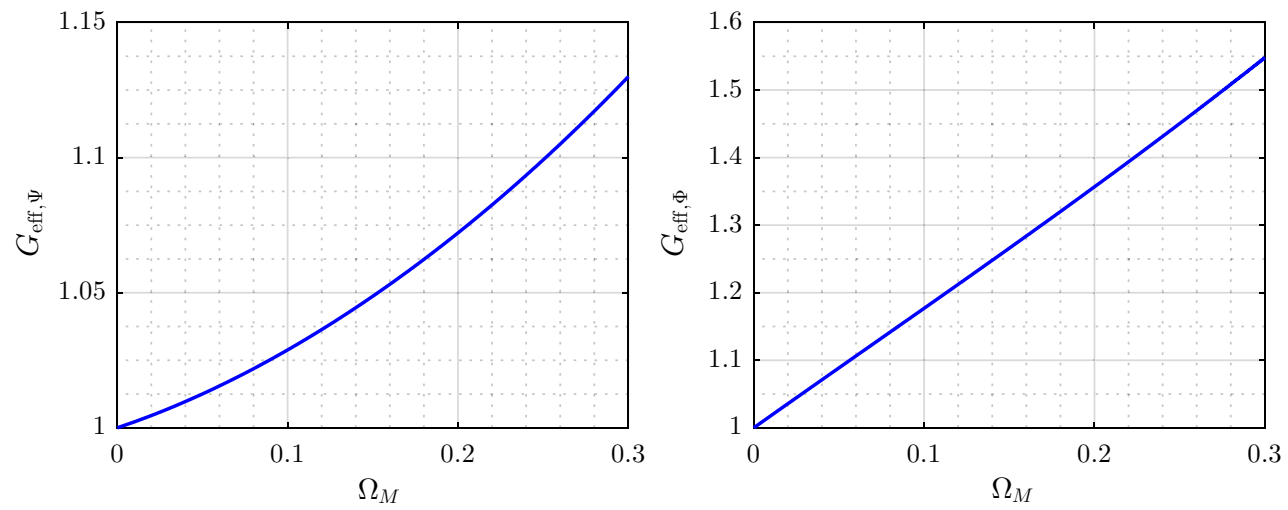

Fig. 2 Plots of $1 / 8 \pi m_{\text {eff, } \Psi}^{2}$ and $1 / 8 \pi m_{\text {eff, } \Phi}^{2}$ respectively versus $\Omega_{M}$. These are proportional to the Newton's constant for each potential respectively. Note that the one for $\Phi$ decreases faster than $\Psi$
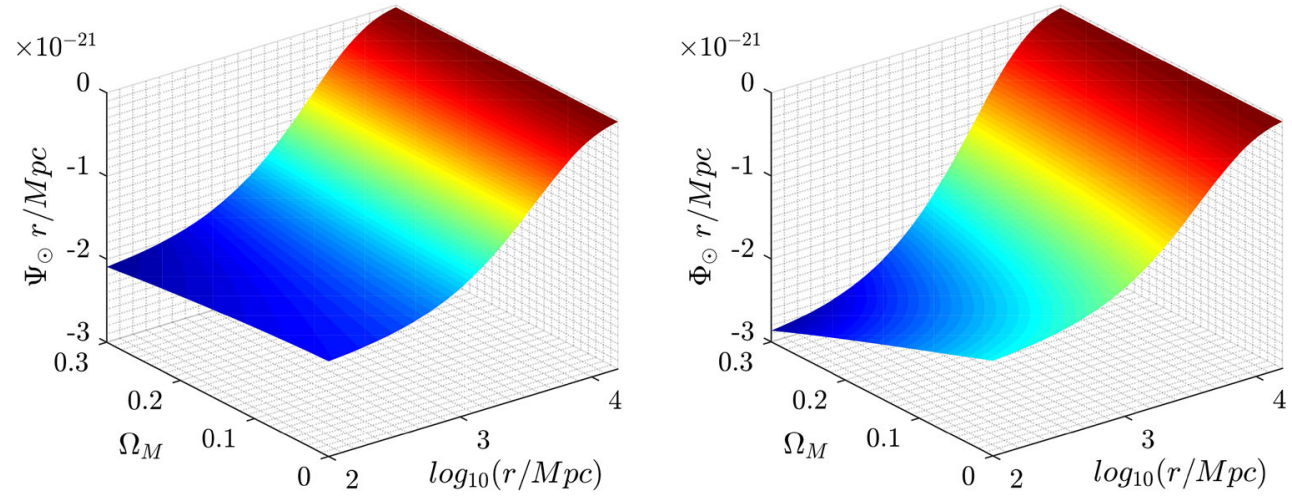

Fig. 3 Plot of $\Psi_{\odot}(r) r / M p c$ and $\Phi_{\odot}(r) r / M p c$ versus $\Omega_{M}$ and $\log (r / M p c)$ for one particle with mass equal to one solar mass

for the potential $\left.\Phi\right|_{\text {one particle }}$. Finally, we depict the potentials in Fig. 3.

We would now like to show that with respect to the universe visible to an observer located at some point $\boldsymbol{x}$, we can actually get rid of the first term in Eq. (35). Indeed, let $N$ be the total number of point sources in Eq. (35) and let $\widetilde{N}$ be the number located within the Hubble horizon radius of an observer located at $\boldsymbol{x}$. Clearly, we may expect that only these $\widetilde{N}$ particles would contribute significantly into Eq. (35). On the other hand, since we should have $N \rightarrow \infty$ in order to obtain a non-vanishing $\bar{\rho}$, it is natural to consider $\widetilde{N} \ll N$. We next split the summations in Eq. (35) into two parts

$\sum_{n=1}^{N \rightarrow \infty}=\sum_{n=1}^{\tilde{N}}+\sum_{n=\tilde{N}+1}^{\infty}$

Since the second summation gets contributions from all particles outside the Hubble horizon of the observer, we can average the potential of this part following [47] and using 
$\bar{\rho}=\sum_{1}^{\infty} m_{n} / V \approx \sum_{\widetilde{N}+1}^{\infty} m_{n} / V$. It is easy to see that this average cancels-out the constant (1/3) term in Eq. (35), leading to

$\left.\Psi\right|_{\text {many particle; average }}=-\frac{1}{8 \pi m_{\mathrm{eff}, \Psi}^{2} a} \sum_{n=1}^{\tilde{N}} \frac{m_{n}}{\left|\boldsymbol{x}-\boldsymbol{x}_{n}\right|} e^{-\frac{\left|\boldsymbol{x}-\boldsymbol{x}_{n}\right|}{\lambda}}$

where "average" in the subscript refers to the aforementioned averaging over sources located outside the observer's Hubble horizon. Note that the above formula has a smooth one particle $(\tilde{N}=1)$ limit, recovering Eq. (37).

\section{Discussion}

At very large length scales of our universe not decoupled from the cosmic expansion, one might expect the gravitational potential to be modified from that of Newton's, as has explicitly been demonstrated for the $\Lambda \mathrm{CDM}$ model in [47]. It is an interesting task to investigate the same for other viable gravity models as well. Being motivated by this, we have investigated the cosmological screening at such large length scales for the phantom braneworld model described in Sect. 2 , with the expectation that the qualitative differences of this model compared to $\Lambda \mathrm{CDM}$ should be maximum at the (super-)horizon scales of our universe. We have presented the equations governing the first order scalar and vector perturbations in Sect. 3. Finally, by ignoring the backreaction effects due to the bulk cosmological constant and the vector perturbation, we have demonstrated analytically and numerically, the behaviour of the two potentials up to the superhorizon length scale in Sect. 4.

It seems to be an interesting task to investigate the tensor perturbation for this model in an early universe scenario. We hope to address this issue in future work.

Acknowledgements SB acknowledges M. Eingorn for mentioning useful references and V. Sahni for discussions on the phantom braneworld model. SRK wishes to acknowledge the ITCP of the University of Crete for a graduate fellowship. TNT wishes to thank CERN-TH for their hospitality during the late stages of this work. SB's work is partially supported by the ISIRD grant 9-298/2017/IITRPR/704.

Open Access This article is distributed under the terms of the Creative Commons Attribution 4.0 International License (http://creativecomm ons.org/licenses/by/4.0/), which permits unrestricted use, distribution, and reproduction in any medium, provided you give appropriate credit to the original author(s) and the source, provide a link to the Creative Commons license, and indicate if changes were made. Funded by SCOAP ${ }^{3}$.

\section{References}

1. P. Brax, C. van de Bruck, A.C. Davis, Brane world cosmology. Rept. Prog. Phys. 67, 2183 (2004). [hep-th/0404011]
2. R. Maartens, K. Koyama, Brane-world gravity. Living Rev. Rel. 13, 5 (2010). arXiv:1004.3962 [hep-th]

3. H. Maeda, N. Dadhich, Matter without matter: Novel KaluzaKlein spacetime in Einstein-Gauss-Bonnet gravity. Phys. Rev. D 75, 044007 (2007). [hep-th/0611188]

4. L. Randall, R. Sundrum, A Large mass hierarchy from a small extra dimension. Phys. Rev. Lett. 83, 3370 (1999). [hep-ph/9905221]

5. L. Randall, R. Sundrum, An alternative to compactification. Phys. Rev. Lett. 83, 4690 (1999). [hep-th/9906064]

6. R. Durrer, P. Kocian, Testing extra dimensions with the binary pulsar. Class. Quant. Gravity 21, 2127 (2004). [hep-th/0305181]

7. M.K. Mak, T. Harko, Can the galactic rotation curves be explained in brane world models? Phys. Rev. D 70, 024010 (2004). [grqc/0404104]

8. S. Pal, S. Bharadwaj, S. Kar, Can extra dimensional effects replace dark matter? Phys. Lett. B 609, 194 (2005). [gr-qc/0409023]

9. T. Harko, K.S. Cheng, Galactic metric, dark radiation, dark pressure and gravitational lensing in brane world models. Astrophys. J. 636, 8 (2005). [astro-ph/0509576]

10. S. Pal, S. Kar, Gravitational lensing in braneworld gravity: formalism and applications. Class. Quant. Gravity 25, 045003 (2008). arXiv:0707.0223 [gr-qc]

11. C.G. Boehmer, T. Harko, Galactic dark matter as a bulk effect on the brane. Class. Quant. Gravity 24, 3191 (2007). arXiv:0705.2496 [gr-qc]

12. T. Harko, K.S. Cheng, The Virial theorem and the dynamics of clusters of galaxies in the brane world models. Phys. Rev. D 76, 044013 (2007). arXiv:0707.1128 [gr-qc]

13. Y. Shtanov, V. Sahni, Bouncing brane worlds. Phys. Lett. B 557, 1 (2003). [gr-qc/0208047]

14. P. Kanti, N. Pappas, K. Zuleta, On the localization of fourdimensional brane-world black holes. Class. Quant. Gravity 30, 235017 (2013). arXiv:1309.7642 [hep-th]

15. P. Kanti, N. Pappas, T. Pappas, On the localisation of fourdimensional brane-world black holes: II The general case. Class. Quant. Gravity 33(1), 015003 (2016). arXiv:1507.02625 [hep-th]

16. A. Nakonieczna, R.M. Nakonieczny, M. Rogatko, Static configurations and evolution of higher dimensional brane-dilaton black hole system. JHEP 1612, 064 (2016). arXiv:1610.01956 [gr-qc]

17. S. Kar, S. Lahiri, S. SenGupta, A note on spherically symmetric, static spacetimes in KannoSoda on-brane gravity. Gen. Rel. Gravity 47(6), 70 (2015). arXiv:1501.00686 [hep-th]

18. K. Yagi, L.C. Stein, Black hole based tests of general relativity. Class. Quant. Gravity 33(5), 054001 (2016). arXiv: 1602.02413 [grqc]

19. S. Chakraborty, S. SenGupta, Strong gravitational lensing — a probe for extra dimensions and Kalb-Ramond field. JCAP 1707(07), 045 (2017). arXiv:1611.06936 [gr-qc]

20. E. Kiritsis, N. Tetradis, T.N. Tomaras, Induced gravity on RS branes. JHEP 0203, 019 (2002). [hep-th/0202037]

21. E. Kiritsis, G. Kofinas, N. Tetradis, T.N. Tomaras, V. Zarikas, Cosmological evolution with brane bulk energy exchange. JHEP 0302, 035 (2003). [hep-th/0207060]

22. G. Kofinas, G. Panotopoulos, T.N. Tomaras, Brane-bulk energy exchange: a model with the present universe as a global attractor. JHEP 0601, 107 (2006). [hep-th/0510207]

23. T. Clifton, P.G. Ferreira, A. Padilla, C. Skordis, Modified gravity and cosmology. Phys. Rep. 513, 1-189 (2012). arXiv:1106.2476

24. H. Collins, B. Holdom, Brane cosmologies without orbifolds. Phys. Rev. D 62, 105009 (2000). [hep-ph/0003173]

25. G.R. Dvali, G. Gabadadze, M. Porrati, 4-D gravity on a brane in 5-D Minkowski space. Phys. Lett. B 485, 208 (2000). [hep-th/0005016]

26. G.R. Dvali, G. Gabadadze, Gravity on a brane in infinite volume extra space. Phys. Rev. D 63, 065007 (2001). [hep-th/0008054]

27. Y.V. Shtanov, On Brane World Cosmology. [hep-th/0005193] 
28. C. Deffayet, Cosmology on a brane in Minkowski bulk. Phys. Lett. B 502, 199 (2001). [hep-th/0010186]

29. C. Deffayet, G.R. Dvali, G. Gabadadze, Accelerated universe from gravity leaking to extra dimensions. Phys. Rev. D 65, 044023 (2002). [astro-ph/0105068]

30. C. Charmousis, R. Gregory, N. Kaloper, A. Padilla, DGP specteroscopy. JHEP 0610, 066 (2006). [hep-th/0604086]

31. D. Gorbunov, K. Koyama, S. Sibiryakov, More on ghosts in DGP model. Phys. Rev. D 73, 044016 (2006). [hep-th/0512097]

32. K. Koyama, Ghosts in the self-accelerating universe. Class. Quant. Gravity 24(24), R231 (2007). arXiv:0709.2399 [hep-th]

33. V. Sahni, Y. Shtanov, Brane world models of dark energy. JCAP 0311, 014 (2003). [astro-ph/0202346]

34. A. Lue, G.D. Starkman, How a brane cosmological constant can trick us into thinking that $w<-1$. Phys. Rev. D 70, 101501 (2004). [astro-ph/0408246]

35. V. Sahni, Y. Shtanov, Did the Universe loiter at high redshifts? Phys. Rev. D 71, 084018 (2005). [astro-ph/0410221]

36. U. Alam, V. Sahni, Confronting braneworld cosmology with supernova data and baryon oscillations. Phys. Rev. D 73, 084024 (2006). [astro-ph/0511473]

37. D. Iakubovskyi, Y. Shtanov, Braneworld Cosmological Solutions and Their Stability. arXiv:0408093 [gr-qc]

38. S. Weinberg, Cosmology (Oxford University Press, Press, 2008)

39. A.V. Viznyuk, Y.V. Shtanov, Scalar cosmological perturbations on the brane. Ukr. J. Phys. 57, 1257 (2012)

40. S. Bag, A. Viznyuk, Y. Shtanov, V. Sahni, Cosmological perturbations on the phantom brane. JCAP 1607(07), 038 (2016). arXiv: 1603.01277 [gr-qc]

41. U. Alam, S. Bag, V. Sahni, Constraining the cosmology of the phantom brane using distance measures. Phys. Rev. D 95(2), 023524 (2017). arXiv:1605.04707 [astro-ph.CO]

42. S. Bhattacharya, S.R. Kousvos, Constraining the phantom braneworld model from cosmic structure sizes. Phys. Rev. D 96(10), 104006 (2017). arXiv:1706.06268 [gr-qc]

43. L. Visinelli, N. Bolis, S. Vagnozzi, Probing extra dimensions with gravitational and electromagnetic signals from compact mergers. arXiv: 1711.06628 [gr-qc]

44. S. Vagnozzi, S. Dhawan, M. Gerbino, K. Freese, A. Goobar, O. Mena, Constraints on the sum of the neutrino masses in dynamical dark energy models with $w(z) \geq-1$ are tighter than those obtained in $\Lambda$ CDM. arXiv:1801.08553 [astro-ph.CO]

45. E. Kiritsis, N. Tetradis, T.N. Tomaras, Induced brane gravity: realizations and limitations. JHEP 0108, 012 (2001). [hep-th/0106050]

46. E. Kiritsis, N. Tetradis, T.N. Tomaras, Induced Gravity and Cosmology on Gravitating Branes. In: Proceedings, Corfu Summer Institute on Elementary Particle Physics (Corfu 2001): Corfu, Greece, August 31-September 20 (2001)
47. M. Eingorn, First-order cosmological perturbations engendered by point-like masses. Astrophys. J. 825, 2 (2016). arXiv:1509.03835

48. M. Eingorn, R. Brilenkov, Perfect fluids with $\omega=$ const as sources of scalar cosmological perturbations. Phys. Dark Univ. 17, 63 (2017). arXiv:1509.08181 [gr-qc]

49. A. Burgazli, A. Zhuk, J. Morais, M. Bouhmadi-Lpez, K. Sravan Kumar, Coupled scalar fields in the late Universe: the mechanical approach and the late cosmic acceleration. JCAP 1609(09), 045 (2016). arXiv:1512.03819 [gr-qc]

50. A. Zhuk, Perfect fluids coupled to inhomogeneities in the late Universe. Gravity Cosmol. 22(2), 159 (2016). arXiv:1601.01939 [grqc]

51. M. Bouhmadi-Lpez, K.S. Kumar, J. Marto, J. Morais, A. Zhuk, $K$ essence model from the mechanical approach point of view: coupled scalar field and the late cosmic acceleration. JCAP 1607(07), 050 (2016). arXiv: 1605.03212 [gr-qc]

52. M. Eingorn, C. Kiefer, A. Zhuk, Scalar and vector perturbations in a universe with discrete and continuous matter sources. JCAP 1609(09), 032 (2016). arXiv:1607.03394 [gr-qc]

53. M. Eingorn, C. Kiefer, A. Zhuk, Cosmic screening of the gravitational interaction. Int. J. Mod. Phys. D 26(12), 1743012 (2017). arXiv: 1711.01759 [gr-qc]

54. O. Hahn, A. Paranjape, General relativistic screening in cosmological simulations. Phys. Rev. D 94(8), 083511 (2016). arXiv:1602.07699 [astro-ph.CO]

55. C. Fidler, T. Tram, C. Rampf, R. Crittenden, K. Koyama, D. Wands, General relativistic weak-field limit and Newtonian N-body simulations. JCAP 1712(12), 022 (2017). arXiv:1708.07769 [astroph.CO]

56. R. Brilenkov, M. Eingorn, Second-order cosmological perturbations engendered by point-like masses. Astrophys. J. 845(2), 153 (2017). arXiv:1703.10282 [gr-qc]

57. B. Wang, Y. Zhang, Second-order cosmological perturbations. I. Produced by scalar-scalar coupling in synchronous gauge. Phys. Rev. D 96(10), 103522 (2017). arXiv:1710.06641 [gr-qc]

58. M. Girardi, A. Biviano, G. Giuricin, F. Mardirossian, M. Mezzetti, Velocity dispersions in galaxy clusters. Astrophys. J. 404, 38 (1993)

59. P.A.R. Ade, [Planck Collaboration], et al. Planck 2013 results. XVI. Cosmological parameters'. Astron. Astrophys. 571, A16 (2014). arXiv:1303.5076 [astro-ph.CO]

60. N.E. Chisari, M. Zaldarriaga, Connection between Newtonian simulations and general relativity. Phys. Rev. D 83, 123505 (2011). arXiv:1101.3555 [astro-ph.CO] 